\title{
PROPOSTA PARA A ESTRUTURA DO SINTAGMA VERBAL CINDIDO: CONCHA v-VP
}

Mário Garcia ${ }^{1}$, UFMG/Poslin

\section{Introdução}

No âmbito da gramática gerativa, o trabalho de Chomsky (1995) assume uma reestrutura no sintagma verbal VP. Agora, o sintagma VP projeta o sintagma externo vP que possui uma função híbrida lexical-funcional por gerar o DP argumento externo em posição de Spec-vP, selecionar o verbo lexical e conter o verbo causativo leve. Esta estrutura é metaforicamente denominada de concha $\mathrm{v}$-VP. Considerando essa nova estrutura, esse texto visa contribuir para uma descrição da origem da proposta da concha v-VP, destacando que a concha v-VP surge a partir do princípio de c-comando assimétrico nas orações com objeto duplo (Larson, 1988) e das evidências da manifestação do verbo causativo nas línguas naturais.

Adotar a proposta v-VP significa que teremos que responder a pelo menos cinco indagações, a saber:

(i) Por que se propõe uma mudança na estrutura de um sintagma verbal simples VP?

(ii) Quais as relações estruturais entre os argumentos e o núcleo que não são codificadas na estrutura VP?

(iii) Há evidências nas línguas naturais para a proposta da concha v-VP?

(iv) Quais são as características do núcleo do novo sintagma, vP?

(v) Como a estrutura $\mathrm{v}$-VP organiza os argumentos e o núcleo dos verbos monotransitivos, ditransitivos e intransitivos (inacusativos e inergativos)?

Para respondermos as questões acima, organizamos este artigo em quatro seções. Na seção 1, apresentamos a discussão originária da concha vVP que se refere ao fato de orações de objeto duplo não captarem o princípio de c-comando assimétrico. Na seção 2, discutimos a organização da concha v-

\footnotetext{
${ }^{1}$ O presente trabalho foi realizado com o apoio do Conselho Nacional de Desenvolvimento Científico e Tecnológico - CNPq - Brasil.
} 
VP nas orações de objeto duplo, destacando que para essa proposta é necessário postularmos um núcleo v com características lexicais, por conter o DP argumento externo, e funcional, por selecionar o verbo lexical por meio do verbo causativo leve. Na seção 3, analisamos a estrutura $\mathrm{v}$-VP nos predicados monotransitivos, ditransitivos (oração dativa e com objeto duplo) e intransitivos (inergativos e inacusativos). E, por fim, na seção 4, apontamos as principais conclusões.

\section{Estrutura VP e o princípio de c-comando assimétrico em orações com verbos ditransitivos}

Dentro do quadro teórico da Gramática Gerativa, a discussão para a elaboração de um VP complexo iniciou-se a partir do questionamento sobre a estrutura sintática de orações com verbos ditransitivos e o princípio de ccomando assimétrico ${ }^{2}$ entre os DPs objetos dessas orações. Barss e Lasnik (1986) observaram que orações com verbos ditransitivos aplicam o princípio de c-comando assimétrico entre os DPs objetos direto e indireto. Para evidenciar a relação de c-comando assimétrico entre esses DPs, os autores verificaram a alternância dativa que consiste na variação entre orações dativas, caracterizadas pela ordem VERBO-OBJETO DIRETO - OBJETO INDIRETO PREPOSICIONADO [V-DPPP], e orações de objeto duplo, que possuem a ordem VERBO - OBJETO INDIRETO OBJETO direto [V-DP-DP].

Dentre as orações observadas, os autores arrolaram as seguintes construções envolvendo o princípio de c-comando assimétrico entre os DPs objetos:

(1a) I showed Mary herself

(1b) *I showed herself Mary

\footnotetext{
${ }^{2}$ A noção de c-comando simétrico é $\alpha$ c-comanda $\beta$ se não domina $\beta$ e todo o $\gamma$ que domina $\alpha$ também $\beta$. De acordo com essa noção, podemos concluir que $\alpha$ e $\beta$ estão num mesmo nível hierárquico. Isso significa que $\alpha$ c-comanda $\beta$ e $\beta$ c-comanda $\alpha$.

Já para o c-comando assimétrico, postula-se que $\alpha$ c-comanda $\beta$, mas $\beta$ não c-comanda $\alpha$, isto é, $\alpha$ e $\beta$ estão em níveis hierárquicos diferentes onde $\alpha$ é superior a $\beta$. (cf.Chomsky, 1995:76, 453-455).
} 
(2a) I gave every worker ${ }_{i}$ his $_{i}$ paycheck

(2b) *I gave its $\mathrm{s}_{\mathrm{i}}$ owner every paycheck $\mathrm{k}_{\mathrm{i}}$

(3a) I showed each man the other's socks

(3b) *I showed the other's friend each man

(4a) I showed no one anything

(4b) *I showed anyone nothing

Observando as orações acima, nota-se que em (1a), o NP "Mary" ccomanda o elemento anafórico "herself"; em (2a) e (3a), os DPs formados com os quantificadores "every" e "each" c-comandam os DPs "his paycheck" e "the other" e, por fim, em (4a), o item negativo "no one" c-comanda o pronome negativo "anything”. Então, com base nesses exemplos, verifica-se o princípio de c-comando assimétrico onde o primeiro D/NP objeto c-comanda o segundo D/NP objeto. Se invertermos a ordem, gerando sentenças onde o segundo $\mathrm{D} / \mathrm{NP}$ c-comande o primeiro $\mathrm{D} / \mathrm{NP}$, teremos como resultados orações agramaticais, conforme mostram os exemplos em (1b), (2b), (3b), (4b).

A relação de c-comando assimétrico entre os DPs objetos traz dificuldades na representação sintática das orações de objeto duplo nas estruturas propostas por Oehrle (1976) e Chomsky (1981) que figuram uma relação de c-comando simétrico entre os argumentos internos, conforme mostram os esquemas abaixo:

(5a) OehrLe (1976):

$$
\begin{array}{lll} 
& \text { VP } & \\
\text { e\#i } & & \\
\mathrm{V} & \mathrm{DP} 1 & \mathrm{DP} 2
\end{array}
$$

(5b) CHOmsky (1981):

$\begin{array}{cccc} & \text { ei } & \text { VP } & \\ & \text { V' } & & \\ \text { ei } & & & \text { DP2 } \\ \text { V } & & \text { DP1 } & \end{array}$


Com relação à proposta de Oehrle (1976), nota-se a ruptura da estrutura binária e da relação de c-comando assimétrico entre os DPs objetos. Já em Chomsky (1981), embora os DPs objetos estejam em níveis diferentes, a relação de c-comando é simétrica, pois os DPs estão numa mesma projeção VP. Assim, as orações arroladas em (1b), (2b), (3b), (4b), segundo a estrutura de Oehrle (1976) e Chomsky (1981), seriam gramaticais.

Nas orações dativas, observa-se somente a relação de c-comando assimétrico entre o DP objeto direto e o PP objeto indireto, conforme se vê nas orações e representações sintáticas seguintes:

(6a) I showed Mary to herself

(6b) *I showed herself to Mary

(7a) I gave every check to $_{\mathrm{i}}$ its $\mathrm{s}_{\mathrm{i}}$ owner

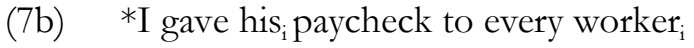

(7a) I sent each boy to the other's parents

(7b) *I sent the other's check to each boy

(8a) I sent no presents to any of the children

(8b) *I sent any of the packages to none of the children

(9a) Oehrle (1976):

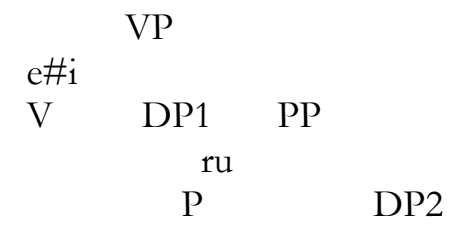

(9b) CHOMsky (1981):

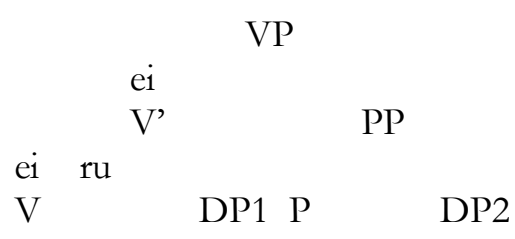

Nota-se que tanto na representação sintática em (9a) quanto na representação em (9b), o primeiro DP objeto sempre c-comanda assimetricamente o segundo DP objeto por estarem em projeções sintáticas 
diferentes. Enquanto DP1 está na projeção VP, o DP2 localiza-se na projeção PP.

Comparando as representações sintáticas das orações de objeto duplo e das orações dativas, propostas por Oehrle (1976) e Chomsky (1981), o questionamento central para a elaboração de um VP complexo está baseado na relação de c-comando assimétrico. Se temos, nas duas orações de verbos ditransitivos, uma relação de c-comando assimétrico entre os argumentos internos, então, por que a representação sintática da oração de objeto duplo não consegue captar a relação de c-comando assimétrico entre os DPs objetos?

\section{Concha v-VP: cisão na estrutura VP e evidências para o sintagma vP}

Vimos na seção (1) que as estruturas ternárias e binárias (Oehrle, 1976) e Chomsky (1981) não conseguem codificar a relação de c-comando assimétrico entre os argumentos internos de orações de objeto duplo. Para adaptar a noção de c-comando assimétrico em construções de objeto duplo, Larson (1988) propõe uma cisão na estrutura VP. Segundo Adger (2004:130), a própria relação de c-comando assimétrico nos impõe a necessidade de postularmos um novo sintagma tanto em orações com objeto dativo, quanto de objeto duplo:

"When we look at double object constructions, we see that there is an asymmetry in c-command relations between goal and object, suggesting a structure with the extra constituent.

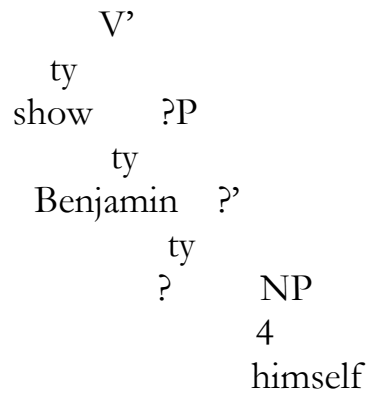


"Emily showed Benjamin bimself in the mirror" [=Emily caused

Benjamin to see bimself in the mirror]"

Para propor esse novo sintagma, precisamos pensar nos traços do núcleo do sintagma ?P. Inicialmente, pode-se assumir que a relação sintática entre o verbo "show" e ?’ contém uma relação semântica de causatividade. Essa relação semântica de causatividade é formalizada nas línguas naturais por meio das orações causativas. A causatividade nas línguas pode ocorrer por processos morfológicos/sintéticos; sintáticos/analíticos e lexicais, conforme verifica-se nos exemplos abaixo:

\section{(i) MORFOLÓGICA/SINTÉTICA}

(a) mudança interna na qualidade da vogal ou mutação da consoante:

$$
\begin{array}{lll}
\text { tiketi } & \text { táikyti } & \text { (Lituano) } \\
\text { ser adequado } & \text { fazer adequação } &
\end{array}
$$

(b) repetição da consoante:
xarab
xarrab
(Árabe)
ser mau
fazer maldade

(c) aumento da duração vocálica

$$
\begin{array}{ll}
\text { mar } & \text { ma:r } \\
\text { morrer } & \text { matar }
\end{array}
$$

(Kashmiri)

(d) mudança tonal

$$
\text { n2 } \mathcal{Y} \text { (ascendente-descendente) n2\& (baixo) (Lahu) }
$$

(e) reduplicação

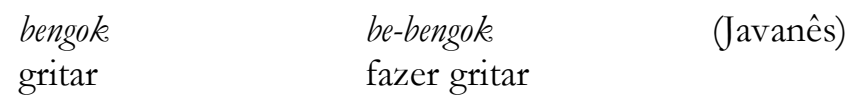

(f) prefixação

$$
\begin{aligned}
& g \star b b a \\
& \text { entrar }
\end{aligned}
$$

$$
\begin{aligned}
& a-g \star b b a \\
& \text { fazer-entrar }
\end{aligned}
$$

(Amharic)

(g) sufixação 
(ii) SinTÁtica/ANALÍTICA

(a) dois verbos que constituem num predicado:

$$
\begin{aligned}
& \text { je ferai manger les gatteaux à Jean } \\
& \text { eu fazer comer os bolos para Jean }
\end{aligned}
$$

(18) bé-k舵-ày-20 $\mathrm{O}$

(b) dois verbos em orações diferentes. Geralmente, o verbo causativo vem na oração principal, enquanto o verbo lexical vem na oração complemento ou em algum tipo de oração subordinada:

$$
\begin{array}{lll}
\text { [imakuipi } & \text { kupi } & \text { Jesus-ya] } \\
\text { mal } & \text { fazer } & \text { Jesús-ERG }
\end{array}
$$

$\begin{array}{lllll}\text { emaputi } & \text { yompa-pi } & \text { makui-ya } & \text { teuren } & \text { (Makushi) } \\ \text { CAUS } & \text { tentar-PASS } & \text { Satanás-ERG } & \text { FRUST } & \end{array}$

"Satanás tentou que Jesus fizesse o mal"

$\begin{array}{lllll}\text { Capi } & \text { te } & {[i-j \tilde{o} t} & n a] & i \text {-to (Canela-kraho) } \\ \text { Capi } & \text { PASS } & \text { eu(S)-dormir } & \text { que } & \text { eu(O)-CAUS } \\ \text { "Capi que me fez dormir" } & & & \end{array}$

(iii) LEXICAL

(a) um verbo pode ser usado em orações não-causativas e causativas:

(23) Mary is knitting Mary is knitting a scarf ( $\mathrm{S}=\mathrm{A})$

"Mary está tricotando" "Mary está tricotando um cachecol"

$$
\begin{array}{ll}
\text { John tripped } & \begin{array}{l}
\text { Mary tripped John } \\
\text { "John tropeçou" }
\end{array}
\end{array}
$$

(b) há dois lexemas verbais, a saber: (i) um expressa intransitividade; (ii) outro expressa a transitividade que corresponde a causatividade:

\begin{tabular}{|c|cc|}
\hline \multicolumn{3}{|c|}{ LÍNGUA: YIMAS - REGIÃO DA PAPUA NOVA GUINÉ } \\
\hline Intransitivos & Transitivos & Tradução \\
mal & tu & "matar" \\
awa & ampu & "queimar" \\
aypu & $\mathrm{t} \gg$ & "deitar" \\
\hline
\end{tabular}




\begin{tabular}{|ccc|}
\hline \multicolumn{3}{|c|}{ LÍNGUA: DYIRBAL-REGĨ̃O DA AUSTRÁLIA } \\
\hline Intransitivos & Transitivos & Tradução \\
mayi & bundi & "sair" \\
gaynyja & bana & "quebrar" \\
jana & jarra & "deitar" \\
\hline & Tabela 2 \\
\hline
\end{tabular}

Causatividade no Português Brasileiro

(i) SINTÁtica

(25) Os PMS fizeram com que os caras pintadas lavassem o rosto

(26) Os grevistas fizeram ver o patrão o quanto estavam sendo radical

(27) O juiz Lalau fez construir um prédio faraônico por uma empresa famosa

(ii) MORFOLÓGICA

(28) A laranja apodreceu/ A umidade apodrecen a laranja-podre $>$ apodrecer

(29) Ex: dolarizar, terceirizar, sarneyzar, fugimorizar, malufar, tucanar, historicizar, computadorizar, informatizar, azulejar, embranquecer, asfaltar, atapetar, adormecer

(iii) LEXICAL

(a) Homônimos

(30) Quanta estação nova de metrô esse Quércia vem construindo [=Quanta estação nova de metrô esse Quércia vem mandando construir]

(31) Cristo morreu na cruzpara nos salvar [=Cristo se fez morrer na cruz para nos salvar]

(32) Abigail caiu no chão só pra impressionar o marido [=Abigail se fez/deixou cair no chão só pra impressionar o marido]

(33) Ele consertou o carro por aquele mecânico de nome maluco [=Ele mandou/fez consertar o carro por aquele mecânico de nome maluco]

(34) Eu almocei os meninos e depois levei eles pra escola [=Eu fiz/dei almoço para os meninos...]

(b) Heterônimo

\begin{tabular}{|l|l|}
\hline \multicolumn{2}{|c|}{ Lexemas verbais } \\
\hline Não-causativos & Causativos \\
Existenciais: & \\
Nascer & Parir \\
Morrer & Matar \\
Desaparecer, sumir & Esconder \\
Epistêmicos & \\
Acreditar, crer & Convencer \\
Aprender & Ensinar \\
Conhecer & Apresentar \\
ver & Mostrar \\
Direcionais & \\
Cair & derrubar \\
\hline
\end{tabular}




\begin{tabular}{|l|l|}
\hline Subir & levantar \\
Entrar & colocar, enfiar \\
Chegar,vir & trazer \\
Ir & levar \\
Sair & tirar, expulsar \\
voltar & devolver \\
\hline \multicolumn{2}{|c|}{ Tabela 3 } \\
\hline
\end{tabular}

Observando os diversos dados sobre causatividade nas línguas, então, pode-se propor um novo sintagma verbal externo vP constituído pelo núcleo $\mathrm{v}$, preenchido pelo verbo causativo que se manifesta da seguinte forma nas línguas naturais:

(i) Semanticamente, isto é, o núcleo é realizado abstratamente, como no caso do inglês e do português;

(ii) Morfologicamente por meio de processos morfológicos internos na palavra;

(iii) Sintaticamente por um verbo auxiliar que pode se constituir numa única unidade ou não com o verbo lexical.

E com relação aos traços, o núcleo $v$ se constitui numa categoria híbrida, isto é, apresenta tanto as características de uma categoria lexical, tendo um traço não-interpretável [+N], quanto de uma categoria funcional, selecionando o verbo lexical.

Com o núcleo v, a oração "Emliy showed Benjamin himself in the mirror" pode ser representada da seguinte maneira:

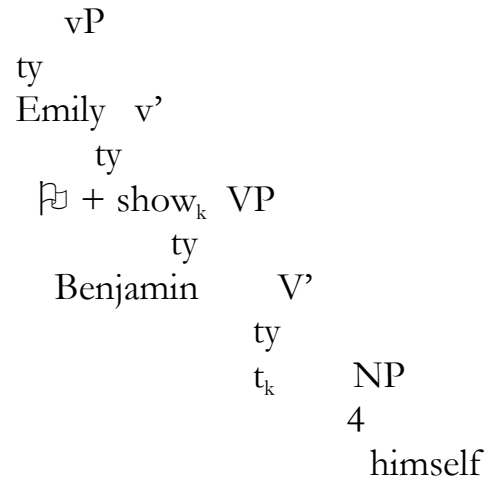

Considerando a configuração (35) e retomando Adger (2004:130), o sintagma ?P passa a ser o sintagma VP que abriga o verbo lexical e os 
argumentos internos e o sintagma vP é formado pelo verbo causativo com o argumento externo.

\section{Concha v-VP nos predicados verbais monotransitivo, ditransitivo, intrasitivo (inergativo e inacusativo)}

Estendo a proposta de Larson (1988), os trabalhos de Chomsky (1995) e Adger (2004) conciliam a concha v-VP com o princípio da Hipótese da Uniformidade na Atribuição dos Papéis- $\theta$ (doravante, UTAH) que estabelece que todas as relações semânticas entre os predicados e os seus argumentos são sempre representadas por uma mesma estrutura sintática na operação merge. ${ }^{3}$ Então, a partir da associação entre estrutura sintática e os papéis- $\theta$, os autores estabelecem as seguintes representações sintáticas para os predicados verbais:

\section{TRANSITIVO}

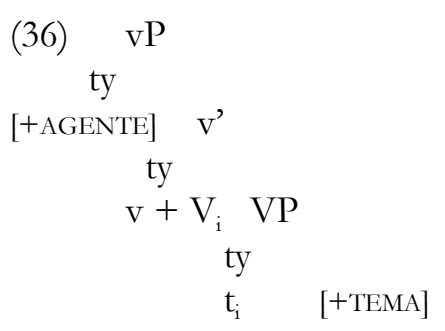

No predicado transitivo, assume-se a seguinte estrutura: um argumento externo [+AGENTE] na posição de Spec-vP; um verbo lexical que se adjunge ao verbo leve; um argumento interno [+TEMA] na posição de complemento do verbo lexical.

\footnotetext{
3 "Uniformity of $\theta$-Assignment Hypothesis (UTAH): identical thematic relationships between predicates and their arguments are represented syntactically by identical structural relationships when items are merged." (Baker, 1985).
} 
$\underline{\text { DITRANSITIVO }}$

ORACִ̃̃O DATIVA

$$
\text { vP }
$$

ty

[+AGENTE] v'

ty
$\mathrm{v}+\mathrm{V}_{\mathrm{i}}$
$\mathrm{ri}$

$\begin{array}{ccc}\text { [+TEMA] } & & \mathrm{V}^{\prime} \\ & \mathrm{ri} & \\ & \mathrm{t}_{\mathrm{i}} & \end{array}$

\section{ORAC̄̃̃O DE OBJETO DUPLO}

vP

ty

[+AGENTE $] \quad \mathrm{v}^{\prime}$

ty

ri

[+ALVO]

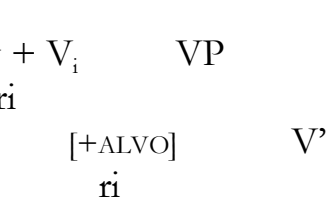

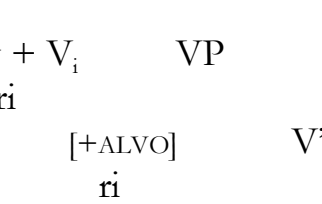

$\mathrm{t}_{\mathrm{i}} \quad$ [+TEMA $]$

O predicado ditransitivo apresenta duas representações sintáticas, sendo uma para a construção dativa e outra para a construção de objeto duplo. Na oração dativa em (37), nota-se o argumento externo [+AGENTE] na posição de Spec vP; o verbo lexical se adjunge ao verbo leve; o argumentos interno [+TEMA], objeto direto, na posição de Spec-VP, e o argumento interno [+ALVO], objeto indireto, na posição de complemento do verbo lexical. Na oração de objeto duplo em (38), observa-se a mesma representação sintática da oração dativa na projeção vP. O que difere é a projeção VP onde os argumentos internos [+ALVO] e [+TEMA] são gerados nas posições de SpecVP e complemento do verbo lexical.

\section{$\underline{\text { INERGATIVO }}$}

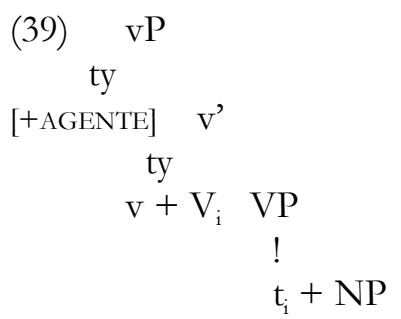

O predicado inergativo possui um argumento externo [+AGENTE] na posição de Spec-vP; um verbo lexical que se adjunge ao verbo leve; um argumento interno que se incorpora ao verbo lexical. 


\section{$\underline{\text { INACUSATIVO }}$}

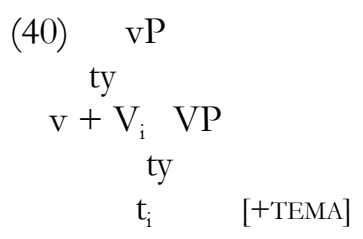

O predicado inacusativo é formado por um verbo lexical que se adjunge ao verbo leve e um argumento interno [+TEMA] na posição de complemento do verbo lexical. É importante ressaltar que Chomsky (1995) considera que os predicados inacusativos não projetam vP, assim, neste caso, teremos somente a projeção VP. Já Adger (2004), trata os predicados inacusativos com a projeção vP, pois se essa projeção é excluída, o princípio UTAH não se realiza. Também, o autor destaca que o núcleo $\mathrm{v}$ dos inacusativos difere dos predicados monotransitivos, ditransitivos e inergativos, por ser um núcleo não-causativo, isto é, não possui um argumento externo com papel- [+AGENTE]. Porém, o verbo lexical move-se para v, pois, semanticamente, o núcleo codifica um acontecimento.

\section{Considerações finais}

Este trabalho examinou a proposta da concha v-VP na gramática gerativa. Vimos que a concha v-VP tem sua origem a partir do princípio de ccomando assimétrico entre os argumentos internos da oração de objeto duplo. Para conseguir explicar o c-comando assimétrico nessas construções, Larson (1988) lança a hipótese de um sintagma verbal externo que abrigue o argumento externo e o verbo causativo leve.

Além das construções de objeto duplo, a concha v-VP encontra evidências nas orações causativas onde o verbo leve pode se manifestar semanticamente, morfologicamente ou sintaticamente. Quando o verbo leve se realiza semanticamente, significa que ele tem uma manifestação abstrata. Já a manifestação morfológica considera os processos morfológicos internos na 
palavra. E a manifestação sintática trata de uma outra unidade sintática, como, por exemplo, o verbo auxiliar, que realiza a causatividade na oração.

$\mathrm{E}$, por fim, verificamos a estrutura $\mathrm{v}-\mathrm{VP}$ nas orações monotransitivas, ditransitivas e intransitivas. Nas monotransitivas, há o argumento externo em Spec-vP e um argumento interno em complemento de VP. Nas ditransitivas, o argumento externo está em Spec-vP, porém os argumentos internos se localizam em posições diferentes. Nas orações dativas, o DP com o papel[+TEMA] é gerado na posição de Spec-VP e o DP com papel- [+ALVO] aparece como complemento de VP. Já nas orações com objeto duplo, o DP [+ALVO] ocorre em Spec-VP e o DP [+TEMA] em complemento de VP. E nas orações intransitivas, os predicados inergativos são compostos pelo argumento externo em Spec-vP e pelo verbo lexical no núcleo de VP, enquanto que os predicados inacusativos têm o argumento interno na posição de complemento de VP e o verbo lexical como núcleo de VP. Com relação à projeção de vP nos predicados inacusativos, Chomsky (1995) adota somente o nível VP, assumindo que os verbos inacusativos projetam Spec-vP, isto é, não possuem um argumento externo, enquanto Adger (2004) considera a projeção de vP, sem Spec-vP. O objetivo do autor ao projetar o nível vP é preservar na configuração sintática o princípio UTHA.

\section{Referências Bibliográficas}

ADGER, David. Core syntax. Oxford: University Press, 2004

BARSS, A. \& LASNIK, H. a note on anaphora and double objects. Linguistic Inquiry, vol. 17, pp. 347-354, 1986.

BITTENCOURT, Vanda de Oliveira. Causativas lexicais no Português do Brasil: perfil morfossintático, semântico e funcional-discursivo. In: DECAT, Maria Beatriz et.al. Aspectos da Gramática do Português. São Paulo: Mercado de Letras, 2001. 
COMRIE, Bernard. Causative verb formation and other verb-deriving morphology. In: SHOPEN, Timothy. Language typology and syntactic description. $2^{\underline{Q}}$ ed. Volume 3. Cambridge: University Press, 1990.

CHOMSKY, Noam. O Programa Minimalista. Tradução: Eduardo Raposo. Lisboa: Caminho, 1995.

DIXON, R. A typology of causatives: form, syntax and meaning. In:

\& AIKHENVALD, Alexandra (orgs.). Changing valency case studies in transitivity. Cambridge: University Press, 2000

LARSON, Richard. On the double object construction. Linguistic Inquiry, vol.19, nํㅜ 3, pp. 335-391, 1988.

MIOTO, C. et. al. Novo Manual de Sintaxe. $2^{a}$ ed. Florianópolis: Insular, 2005.

PAYNE, Thomas. Describing morphosyntax - a guide for field linguists. Cambridge: University Press, 1997. 